\title{
Le petit vignoble de vin liquoreux de Jurançon : un produit et des Hommes innovants?
}

\section{Grégoire Berche}

\section{(2) OpenEdition}

\section{Journals}

Édition électronique

URL : https://journals.openedition.org/geohist/2183

DOI : 10.4000/geohist.2183

ISSN : 2264-2617

Éditeur

Association française de la Revue de géographie historique

Référence électronique

Grégoire Berche, «Le petit vignoble de vin liquoreux de Jurançon : un produit et des Hommes innovants? ", Revue de géographie historique [En ligne], 19-20| 2021, mis en ligne le 20 novembre 2021 consulté le 22 novembre 2022. URL : http://journals.openedition.org/geohist/2183 ; DOI : https:// doi.org/10.4000/geohist.2183

Ce document a été généré automatiquement le 22 novembre 2022.

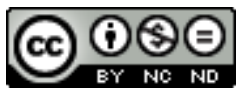

Creative Commons - Attribution - Pas d'Utilisation Commerciale - Pas de Modification 4.0 International - CC BY-NC-ND 4.0

https://creativecommons.org/licenses/by-nc-nd/4.0/ 


\title{
Le petit vignoble de vin liquoreux de Jurançon : un produit et des Hommes innovants?
}

\author{
Grégoire Berche
}

\section{Introduction : Un vignoble issu de la tradition viticole française sur le temps long: Jurançon}

1 Le Jurançonnais est un espace rural viticole (Berche, 2018) situé dans le Béarn, dans le sud de la France (carte 1), au pied des Pyrénées, dans la région administrative Nouvelle Aquitaine. Il se définit par une aire d'appellation spécifique (carte 2) produisant des vins secs (AOC Jurançon sec) et des vins liquoreux (AOC Jurançon). Il a été étudié dans le cadre d'un travail de thèse portant sur trois petits vignobles liquoreux: les vins liquoreux du vignoble de Bergerac (AOC Monbazillac et AOC Monbazillac « Sélection de grains nobles ", AOC Saussignac et AOC Haut-Montravel), AOC vins de paille du Jura et vins liquoreux de Jurançon (AOC Jurançon et AOC Jurançon mention «vendanges tardives" (Berche, 2016). Les enquêtes, menées sous la forme de 26 entretiens semidirectifs à Jurançon entre le 16 et le 27 février 2015, ont ciblé essentiellement des vignerons indépendants, considérés comme les moteurs de l'appellation et potentiellement porteurs d'innovations. Des extraits de ces entretiens seront reproduits en italique dans cet article. Au total, cette étude géographique m'a permis d'appréhender environ 225 hectares d'exploitations indépendantes produisant du vin liquoreux de Jurançon sur 700 hectares de vignes potentiellement aptes à élaborer du vin liquoreux soit environ $80 \%$ du vignoble de vin liquoreux des indépendants de Jurançon. Il existe également une cave coopérative, la cave de Gan-Jurançon, fondée en 1949, qui regroupe 300 viticulteurs, produisant 20000 hectolitres d'AOC Jurançon sur les 37000 hectolitres de vins produits ${ }^{1}$. Toutefois, aucune de mes demandes d'entretien (excepté un coopérateur) n'a été acceptée. 
2 Le vin de Jurançon est associé dans les représentations à un vin avant tout liquoreux (70\% de la production viticole locale) et à la figure du roi Henri IV, représentant l'idéal du Béarnais bon vivant. La cave coopérative de Gan ainsi que de nombreux producteurs indépendants jouent d'ailleurs avec cette image sur leurs étiquettes, la figure d'Henri IV contribuant à forger des discours sur le vin et une origine, dont le légendaire baptême royal au vin de Jurançon le 12 décembre 1553. Ainsi la cave coopérative propose en $2021^{2}$ sept références en Jurançon liquoreux et trois présentent une allusion à Henri IV : le nom d'une cuvée intitulée «la croix du Prince ", une autre, référence incontournable de la cave qui emprunte le nom royal pour sa cuvée "l'apéritif Henri IV » et enfin les fleurs de lys sur l'étiquette de la cuvée «le vieux chai ». Comme l'explique Monsieur P. S. du domaine de Cinquau à Artiguelouve, commune de l'aire d'appellation du Jurançon: " pendant longtemps, les vins produits à travers le monde et qui ne tournaient pas au vinaigre au bout de deux mois étaient des vins sucrés. C'était la seule façon de le conserver à l'époque, y laisser du sucre ou en ajouter ». Il souligne encore : "Si Henri IV a été baptisé selon la légende avec du Jurançon dans la nuit du 12 au 13 décembre 1553, c'était forcément $d u$ vin sucré!» ce qui l'amène à conclure : «On avait un avantage concurrentiel car on avait un vin qui se conservait mieux. Il y avait des périodes où ailleurs il n'y avait pas de vin, nous on en avait » (Entretien du 21/02/15).

3 Le vin liquoreux de Jurançon s'inscrit dès lors dans une temporalité historique assez longue (depuis Henri IV) qui est celle d'une production spécifique, en lien à un territoire particulier, des hommes et des femmes qui le mettent en valeur, et une demande, essentiellement urbaine (Dion, 1959) portée par l'exigence d'une montée en qualité progressive, d'un public, essentiellement français puisque la France représente $90 \%$ des débouchés du Jurançon, qui consomme de manière différente le vin et notamment le vin liquoreux, en fonction des modes et d'attentes nouvelles : consommer moins sucré, associer le vin avec un mets dans le cadre d'une expérience gastronomique, et consommer moins puisque dans l'ensemble, la consommation par habitant diminue depuis 80 ans en France ${ }^{3}$.

4 Dans ces conditions, comment les vignerons produisant du vin liquoreux à Jurançon, notamment les vignerons indépendants, s'adaptent-ils pour continuer à produire et surtout à vendre du vin, et sont-ils condamnés à innover pour exister? 
Carte 1 : Le vignoble de Jurançon dans son contexte régional

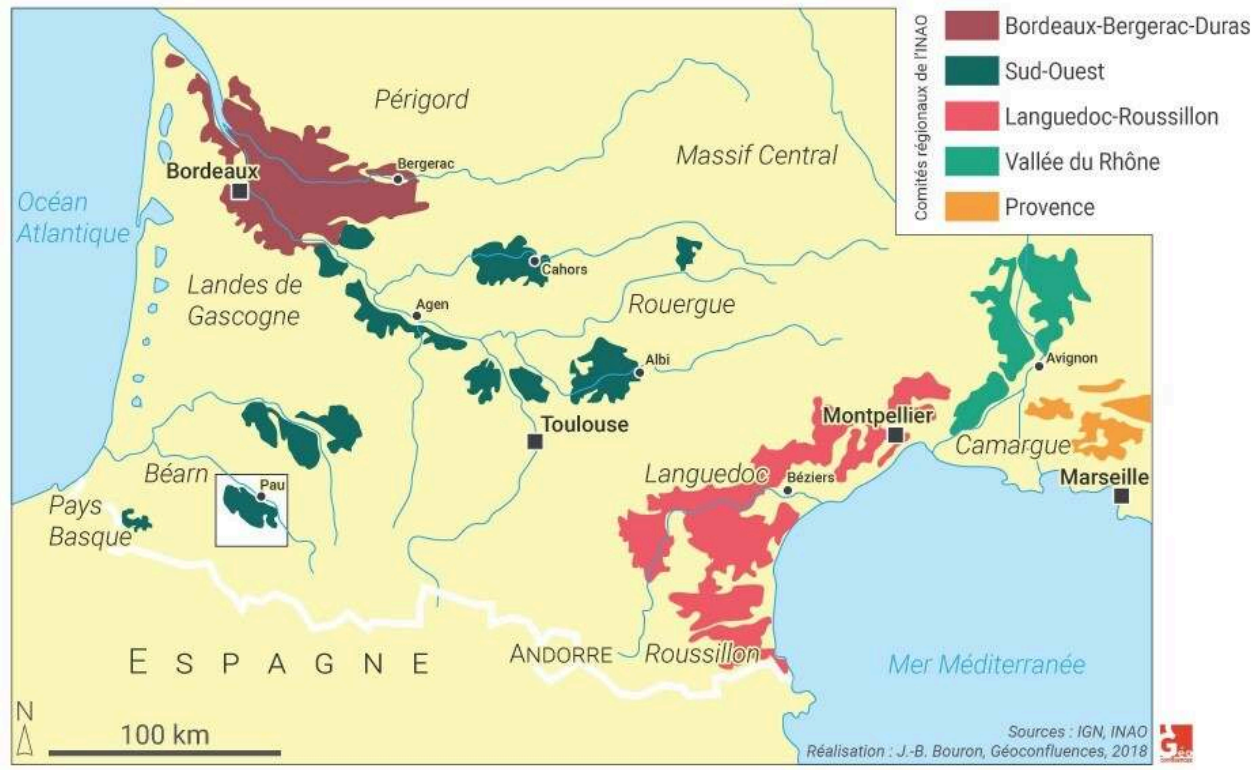

Carte 2 : Localisation de l'aire géographique AOC Jurançon

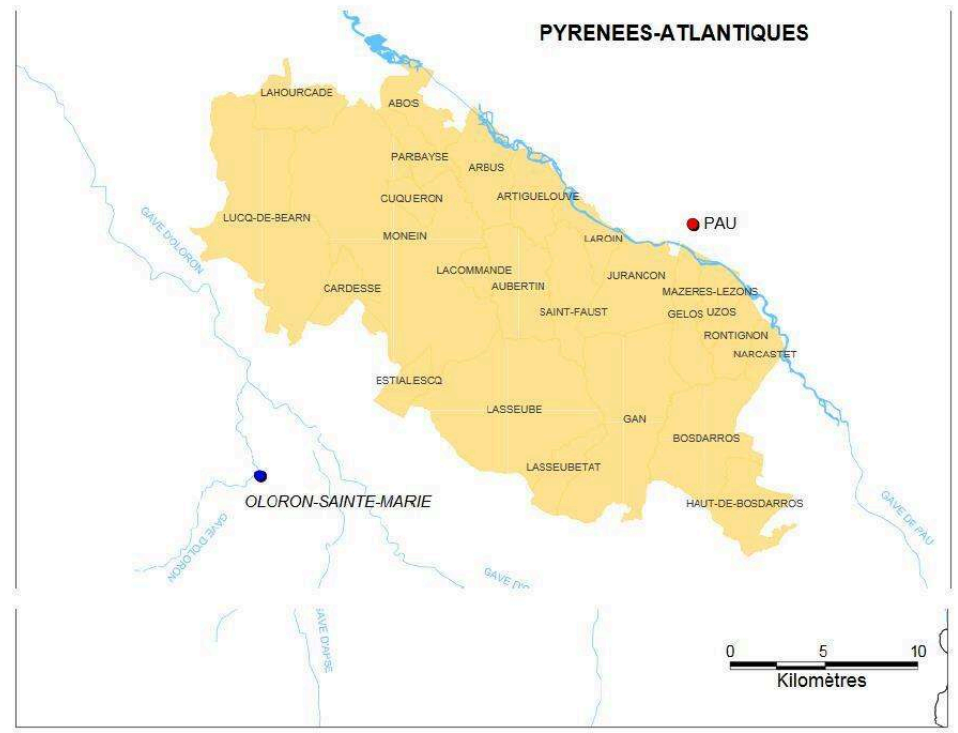

Limites départementales

Réseau hydrographique

Aire géographique

Prefecture

Cours d'eau

AOC Jurançon, Jurançon sec et

- Sous-préfecture

Jurançon vendanges tardives

Source : BDCARTO-IGN, MAPINFO, INAO, 04/2008, Berche, 2016 


\section{Le vin liquoreux à Jurançon : un produit de niche construit dont la vente est reconfigurée par le contexte géoéconomique et géopolitique?}

5 Le vignoble liquoreux de Jurançon, comme l'ensemble du vignoble français, fait face à un contexte géoéconomique et géopolitique nouveau. Ce vin est en effet entré en concurrence avec les vins européens et mondiaux, depuis que le vin est devenu un produit mondialisé. De plus, la majorité du vin se vend aujourd'hui en grandes surfaces, ce qui ne permet pas aux producteurs de dégager des marges suffisantes qui permettraient un réel investissement dans les exploitations, surtout les plus petites, en termes d'équipement (machines, cuveries, dans un contexte d'évolution technique et œnologique du vin), mais aussi en termes d'accueil du consommateur. Des indices, telle la baisse récente du prix du foncier viticole montre un état de crise (le prix moyen d'un hectare de vignes est passé de 52000 euros en moyenne en 2005 à environ 40000 en 2016), d'autant plus que le Jurançonnais produit à $70 \%$ des vins liquoreux, marché qui rencontre des difficultés croissantes, reconnues en 2016 par le président de la Région Nouvelle Aquitaine Alain Rousset (réélu en 2021 à l'occasion des élections régionales) par une expression qui confine à l'euphémisme: "les vins liquoreux traversent une période difficile commercialement ${ }^{4} »$. Le nouveau contexte géopolitique de gestion de la crise sanitaire de la COVID-19 fragilise quant à lui les réseaux de commercialisation (des clients actuels et potentiels) et limite les circulations de tous ordres qui auraient pu bénéficier aux producteurs locaux, d'autant plus que de nombreux producteurs ont opéré une réflexion sur les façons de vendre et l'inscription dans des réseaux de ventes.

6 Certes, comme le rappelle Bernard Pecqueur (Pecqueur, 2001), l'élaboration d'un produit, qu'il soit viticole ou non, s'effectue dans le cadre de la mondialisation qui reconfigure les façons de produire en même temps que les lieux de production. Ainsi, « les évolutions récentes de la mondialisation posent la question de l'extension de la standardisation et de la délocalisation de la production ». Si " la connexion croissante des marchés accentue les dépendances d'un système productif à un autre, les niches productives ont tendance à disparaître ». Mais, par opposition aux produits de masse, un produit de niche s'adresse à une clientèle spécifique d'un segment de marché. Il possède des caractéristiques qui lui permettent de se différencier des autres produits. En l'occurrence, le vin liquoreux est un produit de niche, qui se distingue par sa faible production, et qui se démarque aussi clairement des autres vins par sa couleur, son odeur, ses propriétés organoleptiques, très différentes des autres types de vins, donc par la sensation sucrée et aromatique procurée au consommateur lors de la dégustation. Toutefois, ce qu'on pourrait appeler des " attributs distinctifs " chez Pierre Bourdieu ne passe pas simplement par une distinction sociale. Le vin liquoreux se distingue par son goût, par ses propriétés organoleptiques. Le vin liquoreux suscite également des émotions particulières liées à sa composition chimique, notamment d'ordre physiologique : chaleur et douceur ressenties en bouche, goût plus ou moins prononcé, sucré et/ou acide (dont l'équilibre est d'ailleurs une marque très appréciée des liquoreux de Jurançon, conféré par les propriétés des deux cépages entrant dans sa composition que sont le petit et le gros manseng, qui fait parfois oublier qu'il s'agit d'un liquoreux), mais également d'ordre psychologique, en lien avec des odeurs ou des arômes en bouche qu'on verbalise par des représentations de l'esprit : fruits frais, fruits exotiques, fruits confits, arômes floraux ou qu'on associe à des sensations agréables ou 
désagréables en lien avec son inconscient (Simonnet-Toussaint, 2006), mais plutôt valorisées dans la société occidentale.

7 Un marché de niche, tel que le vin liquoreux de Jurançon, permet souvent d'être confronté à une concurrence moins forte et à des marges éventuellement plus élevées, mais les volumes de ventes potentiels sont mécaniquement plus faibles et limités car les attributs distinctifs du vin (sa couleur, son parfum, sa sucrosité, ses arômes) sont bien souvent difficiles à obtenir. En effet, le coût de fabrication de ce vin est plus élevé : rendements plus faibles ( $40 \mathrm{hl} / \mathrm{ha}$ pour un Jurançon (liquoreux) mais $60 \mathrm{hl} / \mathrm{ha}$ pour un Jurançon sec), donc petites quantités produites, et main-d'œuvre plus nombreuse pour récolter le raisin. On a recours à celle-ci plus longtemps car les vendanges s'étalent dans le temps jusqu'à obtenir des raisins passerillés sur souche, souvent grâce à l'effet de foehn. La production de vin liquoreux demande également une immobilisation d'un nombre plus élevé de cuveries pour les assemblages. L'élevage, souvent plus complexe et plus long que les autres vins, exige des contenants plus nobles (barriques en fûts de chêne neuves ou d'occasion). Pour le cas du vin liquoreux donc, le coût de production est particulièrement élevé, bien qu'il ne soit souvent pas répercuté sur le prix de vente final, obligeant nombre de producteurs à proposer d'autres produits, à l'intégrer à une gamme de vins et ne pas en faire le premier vin de l'exploitation. de vins blancs secs ou de vins rouges, et donc a fortiori de vins liquoreux d'exception qui répondent à des critères de productions que ne peuvent pas s'offrir l'ensemble des producteurs de liquoreux, ne serait-ce qu'en raison de leur coût de production.

9 Mais si les niches peuvent avoir tendance à disparaître d'après B. Pecqueur - ce qui semble être le cas pour le liquoreux face à la progression des autres types de vin que sont le vin rouge, le vin blanc sec et le rosé puisque "dans le monde, sur 100 bouteilles vendues, 90 sont du vin rouge et $10 \mathrm{du}$ vin blanc et sur les 10 bouteilles de vin blanc, 9 sont du vin blanc sec et 1 du vin blanc moelleux ou liquoreux " pour P. S. du domaine de Cinquau (entretien du 21/02/15) - , B. Pecqueur souligne toutefois une nouvelle tendance consécutive au développement de la mondialisation, à savoir le maintien de ces niches : "Pourtant, et ce pourrait être un paradoxe, ces conditions de production et de débouchés des produits et des services valorisent la recherche de ressources nouvelles qui, à l'échelle de systèmes productifs localisés, présentent des caractéristiques spécifiques (c'est-à-dire dédiées à un usage productif particulier et non reproductibles, dans le cas de la spécificité pure) » (page 37, Pecqueur, 2001). La production de vin liquoreux à Jurançon fait partie de ces bienslà : elle n'est guère reproductible et s'inscrit dans des espaces et des sociétés particuliers : c'est donc une niche qui se maintient.

Pour reprendre la pensée de B. Pecqueur, la mondialisation générerait donc des " délocalisations et un réagencement des flux en même temps qu'elle conforterait les régions de production qui se sont spécialisées avant la révolution agricole des années 1950 ", soit celles qui étaient dotées d'un avantage comparatif spatial tel que les acteurs pouvaient y élaborer des produits particuliers, ce qui semble être le cas du Jurançonnais. Ce n'est pas sans rappeler les termes du chapitre $\mathrm{X}$ du cahier des charges de chaque appellation, avec le "lien à la zone géographique " qui convoque systématiquement l'ancrage spatial lié à l'élaboration d'un produit particulier et reconnu par les consommateurs sur le temps long, suggérant la transmission de savoirfaire par les sociétés viticoles locales comme on le constate pour l'appellation Jurançon (Encadré 1). 
$\mathbf{1}^{\circ}$ - Informations sur la zone géographique

a) - « Description des facteurs naturels contribuant au lien

La zone géographique est localisée sur le piémont nord des Pyrénées, en secteur de collines. Le vignoble est relativement disséminé mais il marque le paysage car il est positionné essentiellement sur les hauts de coteaux. [...]

Le climat est marqué par une forte pluviométrie annuelle $(1200 \mathrm{~mm})$ répartie de façon homogène sans saison sèche. L'ensoleillement annuel est moyen (1900 heures). Cependant, le vent du sud chaud et sec, de type foehn, souffle en automne et au printemps ( 1 jour sur 3 en moyenne). Son rôle est fondamental : il assèche l'air et assure une bonne ventilation aussi, il augmente les températures et l'ensoleillement.

Les vignes s'insèrent dans un système de polyculture-élevage traditionnel, et côtoient des bois, des prairies et des cultures. Elles occupent souvent les hauts de coteaux et les pentes les plus fortes, grâce à des plantations en terrasses. La zone géographique s'étend ainsi sur 25 communes situées dans le sud du département des Pyrénées-Atlantiques, au sud et à l'ouest de Pau.

b) - Description des facteurs humains contribuant au lien

Une mosaïque représentant des feuilles de vignes et des grappes de raisins a été retrouvée dans une villa Aquitano-Romaine à Jurançon. Il est cependant très probable que le vignoble ne se soit développé dans la zone géographique qu'au cours du XIV ${ }^{e}$ siècle, période au cours de laquelle l'augmentation de la population entraine un défrichement important et un développement de l'agriculture sur les coteaux partiellement déboisés. La vigne s'installe alors dans des quartiers spécialisés, à proximité des hameaux.

Le $\mathrm{XV}^{\mathrm{e}}$ siècle voit l'augmentation du commerce liée à la création de bourgs importants (Gan, Monein) et surtout au développement de la ville de Pau. En effet, les vicomtes de Béarn s'y installent avec une partie de la bourgeoisie. Ils commencent à acheter des vignes dans les coteaux Jurançonnais voisins de Pau pour satisfaire à la mode qui consistait à boire le vin de sa propre vigne. De plus, le privilège du vin de cru permettait de faire entrer son propre vin en ville avec des droits réduits afin de le commercialiser. Ainsi en 1550, Henri II, le roi de Navarre et sa fille Jeanne d'Albret possèdent des vignes à Jurançon. La place que tiennent la vigne et le vin dans le For de Béarn de 1551 (législation édictée par Henri d'Albret), contrairement aux fors précédents, illustre bien l'importance prise par le vignoble depuis le début de ce siècle. Le cépage " Mansengue » est cité dès le XVI e siècle. Le lien entre le vignoble Jurançonnais et Pau, puis avec les autres villes et régions voisines (Oloron, Lescar, La Vallée d'Ossau), a permis la création de grands domaines viticoles et le développement important des marchés. Ils ont pris une ampleur croissante notamment par l'action des Rois de Navarre, installés à Pau, qui ont promu le vignoble et la consommation des vins de Jurançon à leur cour. A 
cette époque, les vins de Jurançon jouissent d'une grande réputation dans le Béarn mais aussi en France où ils étaient classés parmi les meilleurs vins moelleux. Cette réputation perdure au XVII ${ }^{\text {e }}$ et XVIII ${ }^{\mathrm{e}}$ siècles, période au cours de laquelle le vignoble s'étend, à la suite de l'augmentation de la consommation. Ainsi, en 1640, Marca affirme dans son « Histoire du Béarn » que : « les vins de Jurançon sont d'une bonté exquise qui surpasse les meilleurs de Chalosse et du Bordelais et par conséquent presque toute la France. Les coteaux des lieux voisins de Jurançon comme Gan, Gélos, Saint-Fos et Artiguelouve produisent des vins d'une bonté fort peu différente ». Au XVIII ${ }^{e}$ siècle les cépages " petit manseng, gros manseng, camaralet et courbu » apparaissent dans les écrits des Etats de Béarn. C'est également au XVIII ${ }^{\mathrm{e}}$ siècle que des progrès techniques dans l'élaboration et la conservation des vins permettent d'envisager des exportations à grande échelle. Les Etats du Béarn soutiennent le développement de marchés d'exportation vers la Hollande par le port de Bayonne. Ce marché a pris une importance croissante jusqu'à la fin du XVIII ${ }^{e}$ siècle. La superficie du vignoble commence à régresser à l'arrivée des maladies cryptogamiques et du phylloxera mais surtout au cours des deux guerres mondiales, en passant de 6000 ha à la fin du XIX ${ }^{e}$ siècle, à moins de 600 hectares au milieu du XXe siècle. Le vignoble restant est reconnu en appellation d'origine contrôlée pour les vins blancs moelleux en 1936 et pour les vins blancs secs en 1975. La mention « vendanges tardives » peut compléter le nom de l'appellation d'origine contrôlée depuis 1995. La cave coopérative de Gan a été créée en 1949 et a permis la survie des nombreuses petites exploitations agricoles. Elle produit $60 \%$ des volumes de l'appellation. Une soixantaine de caves particulières produisant du Jurançon sont réparties sur l'ensemble de l'aire. La spécialisation des exploitations vers la monoculture de la vigne, inexistante au début du siècle, devient de plus en plus marquée. La surface du vignoble croît à un rythme régulier depuis une trentaine d'année et occupe en 2010, 1200 hectares environ.

$2^{\circ}$ - Informations sur les caractéristiques du produit

Les vins sont caractérisés par leur vivacité. Cette acidité équilibre l'alcool et les sucres résiduels pour donner des vins sans lourdeur et d'une grande fraîcheur. Jeunes, les arômes, surtout fruités et floraux, dominent généralement puis évoluent souvent vers plus de complexité au vieillissement avec l'apparition d'arômes de fruits secs et d'épices notamment. Les vins peuvent évoluer de quelques années à plusieurs dizaines d'années. Les vins bénéficiant de la mention « vendanges tardives ", marqués par le passerillage prolongé des raisins en automne, présentent une grande richesse en sucres résiduels et des arômes plus intenses. L'acidité élevée soutient également les vins secs et leur confère une grande vivacité, bien équilibrée par beaucoup de gras. Certains vins secs possèdent un potentiel de vieillissement de quelques années, tout en conservant de la fraîcheur ».

Source : https://info.agriculture.gouv.fr/gedei/site/bo-agri/

document_administratif-0ef9d17f-601a-4f08-bc55-aaaea12b1282/telechargement

11 Cette nécessaire transmission intergénérationnelle des savoir-faire dans les vignobles potentiellement aptes à donner des vins liquoreux a engendré une expertise des acteurs locaux qui se sont ainsi spécialisés dans un type de production et ont cherché à 
améliorer " ce " produit " enclin » à être élaboré dans cette région et nulle part ailleurs, le tout fonctionnant comme une rente de situation. C'est ce que l'INAO reconnaîtra en partie lors de l'élaboration des décrets d'Appellation d'Origine Contrôlée comme les " usages locaux, loyaux et constants " (Kuhnholtz-Lordat, 1991), actant l'existence de régions de production, dans le cadre de ce que J. Frayssignes nomme un "ancrage territorial ", qui renvoie à un " processus intentionnel de mobilisation de ressources territoriales » (Frayssignes, 2008). Cet ancrage permet dès lors de définir un "système productif localisé » (Pecqueur, 2001) qu'on peut définir comme un espace aménagé et mis en valeur pour développer une activité économique et produire de la richesse, reposant sur des acteurs multiples qui en permettent le dynamisme. De fait, les systèmes productifs viticoles de vins liquoreux tels que ceux de l'appellation Jurançon se maintiennent, mais non sans évoluer.

Sur le temps long, les acteurs du vignobles liquoreux de Jurançon ont ainsi « composé une offre qui se distingue de la concurrence et par la même pouvant reconquérir des marges de profit. Tout le débat sur les produits agro-alimentaires de qualité et les procédures de labellisation s'affirme sur cette capacité et tend à proposer aux territoires en crise ou en mutation une alternative au productivisme ", et ce, alors que les pays anciennement producteurs procèdent à des campagnes d'arrachage, diminuent leur production et perdent des parts de marché à l'exportation " (Velasco-Graciet, Dieterich, 2008). Cela se double pour les acteurs des vignobles liquoreux du sud-ouest notamment, d'une véritable mutation territoriale à la fin du $\mathrm{XX}^{\mathrm{e}}$ siècle. C'est le « tournant » qualitatif (Strang, 1997), qui, pour la partie septentrionale et occidentale du Sud-Ouest français viticole, depuis Bordeaux jusqu'aux confins du pays basque, a conduit à la généralisation des vendanges manuelles en remplacement des vendanges mécaniques qui avaient conduit à des crises de surproduction certaines années exceptionnelles ou plus régulièrement à des dérives. En effet, la qualité des moûts obtenus avec la vendange mécanique nécessitait l'ajout de sucre qui lui-même nécessitait l'ajout de souffre pour stabiliser le vin et éviter qu'il ne reparte en fermentation, et les quantités de souffre étaient parfois telles qu'il fallait rajouter de quoi neutraliser ces mêmes excès de souffre. Ces pratiques ont été confirmées dans le Bergeracois par exemple (Berche, 2016) mais dans une moindre mesure à Jurançon. En effet, le tournant qualitatif pris par les vignerons du Jurançonnais a semble-t-il été plus ancien que les autres vignobles liquoreux du Sud-Ouest puisqu'en raison des fortes pentes présentes dans ce vignoble, les vendanges se réalisent depuis longtemps nécessairement à la main, le tracteur ne pouvant que très rarement accéder aux terrains pentus, mais il a été plus long à se mettre en place comme le rappelle Monsieur P. S., vigneron d'Artiguelouve désormais à la retraite : "Au milieu des Trente Glorieuses, c'est l'ouverture du marché européen. Les jeunes sont allés à l'école, ont appris que les cuves en béton et en inox étaient certes moins jolies, mais permettaient de vinifier bien mieux. Les jeunes reviennent chez eux en disant qu'il faudrait se moderniser ! Et ça a bougé ! On a alors associé à cela des méthodes de production de froid. On a pu ainsi faire du Jurançon (liquoreux) sans mettre du souffre. Il y a eu de nouveaux échanges musclés mais ça a passé. On a eu des subventions pour s'équiper, notamment des cuves avec serpentins à l'intérieur puis à l'extérieur pour éviter les accidents et les contaminations. On s'est également équipé en centrales génératrices de froid. Cela a eu lieu dans le milieu des années 1980 ; entre 1965 et 1985 on a appris à faire du Jurançon moelleux (liquoreux) pour éviter que le vin ne reparte en 
fermentation. Désormais, les buveurs de Jurançon moelleux (liquoreux) n'ont plus mal à la tête » (Entretien du 21/02/15).

Le système productif localisé qui s'est donc constitué autour du vignoble de vins liquoreux de Jurançon nécessite donc de travailler sur le modèle spatial qu'est le territoire (le bornage symbolique par une société d'une portion de l'espace terrestre qu'on peut alors qualifier d'appropriée), mais qui est peut-être vue pour l'économiste comme un élément marketing pour vendre et se vendre. Il s'agira donc de voir en quoi les acteurs produisant du vin liquoreux à Jurançon innovent pour se maintenir voire se développer, dans un contexte inédit, marqué par la mondialisation de l'économie, et en quoi la réflexion sur la temporalité alimente ses réflexions, la production territoriale s'inscrivant dans le temps long. Finalement, existe-t-il un modèle territorial quant à la production (et la vente) de vin liquoreux à Jurançon? Pour y répondre, on se propose maintenant d'interroger l'échelle de l'exploitation à travers le groupe des exploitants indépendants.

\section{Des producteurs indépendants qui segmentent leur production et partent à la recherche de ce qu'est un produit de qualité pour mieux vendre}

Il existe trois appellations de vin blanc "Jurançon" telles que mentionnées dans le cahier des charges (Figure 1).

Figure 1 : Appellations AOC Jurançon et leurs caractéristiques œnologiques

a)- Les richesses en sucre des raisins et les titres alcoométriques volumiques naturels répondent aux caractéristiques suivantes :

\begin{tabular}{|c|c|c|}
\hline $\begin{array}{c}\text { APPELLATION D’ORIGINE } \\
\text { CONTROLEE, MENTION }\end{array}$ & $\begin{array}{c}\text { RICHESSE MINIMALE EN } \\
\text { SUCRE DES RAISINS } \\
\text { (en grammes par litre de moût) }\end{array}$ & $\begin{array}{c}\text { TITRE ALCOOMÉTRIQUE } \\
\text { VOLUMIQUE NATUREL MI- } \\
\text { NIMUM }\end{array}$ \\
\hline $\begin{array}{c}\text { AOC « Jurançon » suivie de la } \\
\text { mention « sec » }\end{array}$ & 187 & $11,50 \%$ \\
\hline AOC « Jurançon » & $\begin{array}{c}\text { - cépage petit manseng B : } 247 \\
\text { - autres cépages : } 230\end{array}$ & $14 \%$ \\
\hline $\begin{array}{c}\text { AOC « Jurançon » complétée de la } \\
\text { mention « vendanges tardives » }\end{array}$ & 281 & $17 \%$ \\
\hline
\end{tabular}

Source : https://info.agriculture.gouv.fr/gedei/site/bo-agri/

document_administratif-0ef9d17f-601a-4f08-bc55-aaaea12b1282/telechargement

Lors des enquêtes, on a en effet pu constater que les vignerons utilisent finalement peu la mention «Vendanges Tardives» (VT). A la question " Pourquoi les vignerons n'utilisent pas davantage l'appellation Vendanges Tardives?", voici ce que répond A. V., vigneron indépendant à Aubertin : "Le débat n'a pas lieu d'être. La question à se poser c'est "l'image du Jurançon passe-t-elle par les vendanges tardives ou pas ? "La réponse est oui, pas uniquement, mais elle passe par là. Cela joue. Ce qui fait l'image c'est l'acidité : ça en fait un liquoreux à part. Des vins pointus en attaque et ronds en finale. Le sucre prend ensuite le dessus. C'est la fraîcheur du Jurançon qui fait qu'on ne peut pas le classer dans les vins de dessert. Il n'y a que le Jurançon qui peut amener aussi loin dans le temps la fraîcheur et le côté sucre de la " vendange tardive » " (Entretien du 19/02/15). Le positionnement qualitatif du Jurançon 
passe donc selon A. V. par un faisceau de réalités dont la VT, mais qui vient conforter ce positionnement et non l'impulser.

On peut mettre en regard l'avis d'un autre vigneron de l'appellation, V. M., propriétaire d'une exploitation de 4.7 hectares dans la commune de Lahourcarde. Ce dernier est convaincu de la nécessité d'un positionnement « grand cru » du vin de Jurançon et des moyens qu'il met en œuvre pour y parvenir :

"Je produits des vins haut de gamme, avec de fortes densités, de l'ordre de 8000 pieds à l'hectare alors que la norme ici est de 4000 hectares. Mon positionnement a été réfléchi en 2005. [...] Je laisse moins de bourgeons, moins de grappes, de façon à mieux nourrir le raisin. Mes vignes sont basses. Si je les mets trop haut, je génère de l'ombre et du coup la photosynthèse ne s'effectue pas correctement. J'ai appris cela à Libourne pendant deux ans, puis à Blanquefort pendant 2 ans. Je mène également une réflexion sur la taille, avec la gestion de l'équilibre de ma sève. Je fais venir pour cela des tailleurs, avec un nombre d'hommes à l'hectare très important. Je suis plutôt sur des gammes aromatiques, avec des terpènes (arômes de fruits confits) et des thiols (arômes de fruits frais). Je vendange le plus tard possible, avec une fermentation et un élevage en barrique 12 mois, puis cuve inox 8-9 mois, pour le vieillissement puis mise en bouteille. Ma bouteille est à 16.5 euros prix cave. En CHR ${ }^{5}$, je vends ma bouteille à 8.25 HT et je rajoute la TVA. Je dois payer mes étiquettes, mes bouchons, mes capsules. Les restaurateurs multiplient quant à eux le prix de ma bouteille par 4 ou par 5 sachant que je leur vends à 12.30 euros. Je dénonce le manque de cohérence entre les restaurateurs et le prix appliqué sur le vin. Je compte sur le bouche à oreille, les revendeurs. J'ai un commercial. Il a fallu intégrer un réseau. Mais les rotations sont longues : j'ai un stock et je ne me voyais pas du tout faire avec. Le reste a donc été mis à Castel (un important négociant) à Monein. Cela me permet d'avoir une trésorerie. Le prix moyen est de 3 euros le litre de moût... Mais j'ai vendangé le matin, j'apporte et je n'ai pas de contrainte. Je ne fais pas de salons, c'est cher. Je compte sur les revues d'abord, car ça rentre dans une politique de qualité. Pour les gens, Jurançon c'est le Jura! Du coup, 16.5 euros c'est cher ! Un salon ça me coûte 1500 euros HT plus la location du matériel plus le camion plus l'hébergement plus le restaurant. C'est une journée à perte. Je préfère me faire connaître. Le fait de rentrer dans les belles tables permet de me justifier. Les sommeliers me font confiance. Il y a aussi le système des médailles, avec le Guide Hachette. Mais on n'est pas égaux face aux concours. La cave coopérative de Gan en passe huit, je pense au concours agricole de Paris. Le bio c'est intéressant, mais on a un climat humide, de l'ordre de $1500 \mathrm{~mm} / \mathrm{an}$. On accentue le phénomène de l'évaporation lors des orages d'été. Si on laisse $30 \mathrm{~cm}$ d'herbe, l'humidité devient très importante. Il faut donc que sous le rang, ce soit propre. Je désherbe très peu. Le mildiou je le contiens avec un produit de synthèse et l'oïdium, je le contiens avec du souffre, qui est un produit homologué dans la culture bio. J'ébourgeonne, c'est presque prophylactique, pour limiter les risques de pourriture. Cela prend du temps mais je n'utilise pas d'insecticide. Mais j'ai de plus en plus de cicadelles, ce qui perturbe la photosynthèse. J'utilise de l'argile bio mais cela a ses limites. Par rapport aux autres bios, cela a des incidences économiques. Cela un impact sur mes rendements. En Alsace, où on est très bio, les années pluvieuses cela pose de gros problèmes. Nous on a le problème des montagnes. Économiquement dans le secteur, c'est difficile, Lacq, ce n'est plus rien. Il y a les produits pharmaceutiques. Pour ma part, j'ai fait une sofergie ${ }^{6}$ » (Entretien du 19/02/15). 
" J'ai la volonté de me positionner en créant une marque, mais je ne trouve pas nécessaire d'avoir une agence de consulting. Ce n'est pas la peine selon moi car la marque est là. Même en faisant du mono-cépage, on a une typicité. Il faut attaquer sur la communication autour de Jurançon. Tariquet a créé une marque. La cave coopérative pèse lourd et est très fermée. Castel peut exister en achetant les moûts un peu plus chers. Les vendanges tardives, il y a plus de contrôle, il faut par exemple des vignes qui doivent avoir 5 ans. Il faut vivre de notre produit. Il ne faut pas perdre une vente. Je fais un sec vendangé en novembre donc je me positionne différemment. Je ne veux pas faire un sec de type Côtes de Gascogne, je ne peux pas m'aligner. On est sur un autre produit. Les vins thiolés, c'est bien pour s'initier au vin. Cette clientèle jeune, on va la récupérer. Il faut les attirer en leur proposant quelque chose de différent. Ce n'est pas possible pour les gros de ne faire que des vendanges tardives. Il faut du haut de gamme. Créons une appellation Jurançon grand cru, mais qui ne soit pas une appellation, mais une marque, avec des critères d'exposition, et en $100 \%$ petit manseng. Ayons un budget commun sans forcément favoriser ce vin par rapport aux autres, faisons-le connaître en France, ce n'est pas la peine de le vendre en Chine... Les anciens du Jurançon ont réagi étrangement à ma proposition! » (Entretien du 19/02/15).

Ces deux types de segmentation qui recherchent finalement la reconnaissance institutionnelle du travail particulier d'un espace de l'appellation, le cru, ou un savoirfaire vitivinicole précis, la réalisation de Vendanges Tardives, continuent d'interroger ce qu'est un vin de qualité et donc un produit de qualité. Ceci constitue selon nous le moteur de l'innovation dans ce petit vignoble liquoreux qu'est Jurançon. La toute fin de l'entretien avec A. V. permet de poser un regard lucide sur la définition d'un produit de qualité et les moyens d'y parvenir :

« Le bio est absolument nécessaire. Pour moi, par pour la bonne image que cela apporte, pas tellement. C'est par conviction. Les clients, qu'est-ce qu'ils recherchent? Un produit de qualité.

1) la qualité c'est d'abord l'image connue et reconnue. C'est un produit dont on parle. Ce n'est pas forcément faire du bio à la vigne. Mais c'est celui qui va essayer d'avoir le raisin le mieux constitué et le plus sain possible.

2) la diversité, l'originalité : on fait un rouge, on gagne des clients pour avoir ce rouge. C'est un bon complément de gamme. On apporte les mêmes soins aux parcelles.

3) la curiosité. Le cadre : par un lieu que l'on s'approprie » (Entretien du 19/02/15).

Un vin innovant s'apparente, d'après les enquêtes menées auprès des vignerons indépendants en 2015, à un vin de la meilleure qualité possible. Son élaboration passe donc par un acteur qui tente de produire et de concevoir le meilleur ingrédient possible pour réaliser ce produit. C'est donc nécessairement sans référentiel : toutes les voies sont bonnes selon ce vigneron pour obtenir ce meilleur ingrédient. Le produit de qualité est aussi fonction du fabriquant qui pense qu'il fait le meilleur possible : il doit le prouver par des méthodes à expliquer au consommateur pour les faire reconnaître par ce même consommateur. Cette communication se fait idéalement par l'image de l'appellation, mais peut tout autant aussi passer par une simple communication sur l'étiquette ou un échange verbalisé sur un salon, bref, un discours sur le produit, quel qu'il soit. Un produit de qualité ne se distingue donc pas des autres pour lui-même, mais par le fait que le consommateur reconnait que le fabricant fournit des efforts permanents pour obtenir chaque année un meilleur résultat, ou tout au moins, le meilleur qu'il puisse faire en fonction des conditions qui ont été les siennes. C'est donc 
davantage l'identification d'une démarche de production et l'explicitation de la démarche qui permettent de qualifier un produit de qualité, d'où la limite des labels de qualité qui ne sont pas fondés sur une démarche (nécessairement évolutive) mais des marqueurs géographiques figés comme l'origine (AOC), ou des critères de qualité selon des cahiers des charges (dont les produits biologiques ou Label Rouge par exemple). En d'autres termes, si l'AOC garantit une origine, elle ne garantit pas la bonne conscience du fabricant inscrit dans l'AOC. C'est aussi pour cela que se développent les circuitscourts. La proximité entre acheteur et vendeur est aussi une proximité personnelle : en réduisant la distance, physique et mentale, avec le producteur, on peut discuter du produit élaboré et le vendeur peut communiquer plus facilement à l'acheteur les efforts nouveaux déployés pour fournir un meilleur produit et entretenir son " capital de vente ». La conversion à l'agriculture biologique permet souvent de rendre davantage visible cette démarche du " mieux faire » car elle montre un souci d'évolution vers d'autres pratiques, voire constitue un tournant pour l'exploitation, d'où le foisonnement des labels reconnaissent un travail particulier du vigneron, que ce soit à « l'amont » ou à « l'aval » de l'exploitation. Néanmoins, elle n'est pas forcément la norme.

\section{Conclusion}

Plus qu'un "produit de niche» dont on chercherait à préserver l'identité ou l'existence, les producteurs de liquoreux de Jurançon, notamment les indépendants, innovent en proposant une réflexion sur ce que doit être un produit de qualité, répondant ainsi à une attente forte des consommateurs, dans le contexte de la mondialisation et des effets de celle-ci. Si le discours sur le produit de qualité n'est pas nécessairement le même, aux niveaux de l'appellation et des producteurs, celui-ci semble désormais une nécessité pour continuer d'exister aux yeux des consommateurs et vendre : réaffirmer l'ancrage historique, segmenter la production, définir un grand cru, convertir en bio, mener des démarches environnementales, s'inscrire dans des réseaux de vente avec d'autres producteurs, tout semble possible mais aussi nécessaire. C'est finalement le fait d'avoir opéré l'ensemble de ces réflexions sur ce qu'est un produit de qualité à élaborer par la grande majorité des producteurs, cave coopérative et vignerons indépendants, qui constitue une innovation majeure dans ce petit vignoble qui permet une certaine résilience du vin liquoreux de Jurançon.

\section{BIBLIOGRAPHIE}

Berche G., 2018, « Les campagnes viticoles du Jurançonnais : dynamiques des vignerons indépendants au sein d'un système productif en recomposition », Géoconfluences, juin 2018.

Berche G., 2016, Les vins liquoreux, un produit de terroir en marge dans la hiérarchie vitivinicole française : étude comparée des petits vignobles de vins liquoreux de Bergerac, Jurançon, Jura et Corrèze. 
Thèse de doctorat en géographie réalisée sous la direction de Monique Poulot, Université de Paris-Ouest la Défense, 582 pages.

Dion R., 1959 (rééd. 1993). Histoire de la vigne et du vin en France des origines au XIXe siècle, Flammarion, Paris, 768 pages.

Frayssignes J., 2008, « Démarches de qualité et développement territorial : quels apports pour la géographie rurale ? L'exemple des AOC fromagères françaises », Géocarrefour.

Kuhnolz-Lordat G., 1991. La genèse des appellations d'origine des vins, coll. Avenir œnologie, Chaintré, 148 pages.

Pecqueur B., 2001, «Qualité et développement territorial : l'hypothèse du panier de biens et de services territorialisés », Economie rurale, numéro 261, p. 37-49.

Simonnet-Toussaint C., 2006, Le vin sur le divan, des représentations sociales aux représentations intimes, Féret, Bordeaux, 152 pages.

Strang P., 1997 (traduit de l'anglais par S. Valléjo), Vins du Sud-Ouest, éditions du Rouergue, Rodez, 347 pages.

Velasco-Graciet H., Dietrich F., 2008, « La géopolitique du vin : vins du terroir, vins du nouveau monde » (compte-rendu de conférence), L'avenue, le 24 avril 2008.

\section{NOTES}

1. Site de la cave coopérative de Gan, https://www.cavedejurancon.com/la-cave/qui-sommesnous.html, consulté le 20 août 2021.

2. Site de la cave coopérative de Gan, https://www.cavedejurancon.com/boutique/famille/ juran\%C3\%A7on-doux.html, consulté le 3 août 2021.

3. La consommation de vin en France atteignait en effet $47,8 \mathrm{l} / \mathrm{an} / \mathrm{hab}$ en 2005 , $78 \mathrm{l} / \mathrm{an} / \mathrm{hab}$ en 2001, $91 \mathrm{l} / \mathrm{an} / \mathrm{hab}$ en $1990,123 \mathrm{l} / \mathrm{an} / \mathrm{hab}$ en $1980,143 \mathrm{l} / \mathrm{an} / \mathrm{hab}$ en 1970 et $174 \mathrm{l} / \mathrm{an} / \mathrm{hab}$ en 1960 d'après l'INSEE.

4. Magazine Terre de Vins numéro 40, mars-avril 2016.

5. Réseau de Cavistes, Hôtels et Restauration

6. Le vigneron a en effet fait installer des panneaux photovoltaïques qui lui permettent de faire fonctionner son bâtiment, de revendre l'électricité à EDF et ainsi dégager un complément de revenu.

\section{RÉSUMÉS}

Le vignoble liquoreux de Jurançon constitue un petit vignoble. Il s'est construit sur le temps long, et les producteurs, notamment les vignerons indépendants, cherchent à s'adapter aux évolutions économiques et sociales $\mathrm{du} \mathrm{XX}^{\mathrm{e}}$ siècle et $\mathrm{du}$ début $\mathrm{du} \mathrm{XXI}^{\mathrm{e}}$ siècle notamment pour continuer à élaborer des vins qu'on pourrait qualifier de «produits de niche». Mais, en réalité, pour continuer à exister et à vendre du vin, les producteurs innovent en opérant une réflexion et un discours sur ce qu'est un produit de qualité. 
The sweet wine vineyard of Jurançon is a small vineyard. It has been built over a long period of time, and producers, particularly independent winemakers, are trying to adapt to the economic and social developments of the 20th century and of the beginning of the 21st century, in particular to continue to develop wines that could be described as "niche products". But in reality, in order to continue to exist and to sell wine, producers innovate by reflecting and giving a discourse on what a quality product is.

\section{INDEX}

Keywords : Jurançon, sweet wine, independent winemakers, innovating, sell wine, quality product

Mots-clés : Jurançon, vin liquoreux, producteur indépendant, innovation, vente, produit de qualité

\section{AUTEUR}

\section{GRÉGOIRE BERCHE}

Université Paris Ouest la Défense, LAVUE, 200 Boulevard de la République 92000 Nanterre

gregoire.berche@wanadoo.fr 